\title{
Clinical features, diagnostic challenges, and management strategies in checkpoint inhibitor- related pneumonitis
}

REVIEW

This article was published in the following Dove Press journal:

Cancer Management and Research

14 June 2017

Number of times this article has been viewed

\author{
Sarah Chuzi' \\ Fabio Tavora ${ }^{2}$ \\ Marcelo $\mathrm{Cruz}^{3}$ \\ Ricardo Costa ${ }^{3}$ \\ Young Kwang Chae ${ }^{3}$ \\ Benedito A Carneiro 3 \\ Francis J Giles ${ }^{3}$ \\ 'Department of Medicine, Feinberg \\ School of Medicine, Northwestern \\ University, Chicago, IL, USA; ${ }^{2}$ Argos \\ Laboratory, Messejana Heart and \\ Lung Hospital, Fortaleza, Brazil; \\ ${ }^{3}$ Developmental Therapeutics \\ Program, Division of Hematology/ \\ Oncology, Feinberg School of \\ Medicine, Northwestern University, \\ Chicago, IL, USA
}

\begin{abstract}
Immune checkpoint inhibitors, including cytotoxic T-lymphocyte antigen 4 (CTLA-4) and programmed cell death-1 (PD-1) inhibitors, represent an effective treatment modality for multiple malignancies. Despite the exciting clinical benefits, checkpoint inhibition is associated with a series of immune-related adverse events (irAEs), many of which can be life-threatening and result in significant treatment delays. Pneumonitis is an adverse event of special interest as it led to treatment-related deaths in early clinical trials. This review summarizes the incidence of pneumonitis during treatment with the different checkpoint inhibitors and discusses the prognostic significance of tumor type. The wide range of clinical, radiographic, and histologic characteristics of checkpoint inhibitor-related pneumonitis is reviewed and followed by guidance on the different management strategies.
\end{abstract}

Keywords: immune checkpoint inhibitors, pneumonitis, anti-PD-1, anti-CTLA-4, immunerelated adverse event

\section{Introduction}

The introduction of immune checkpoint inhibitors to clinical practice has changed the landscape of cancer treatment. By targeting cytotoxic T-lymphocyte antigen 4 (CTLA-4) and programmed cell death-1 (PD-1), these medications block negative regulators of T-cell function and enhance antitumor immune activity. ${ }^{1}$ Remarkable tumor responses to these agents have been documented across various malignancies, leading to their approval by the US Food and Drug Administration (FDA) in a growing number of cancers. Ipilimumab (anti-CTLA-4) is approved for the treatment of metastatic melanoma, ${ }^{2}$ and anti-PD-1 agents (i.e., pembrolizumab and nivolumab) are approved for advanced non-small cell lung cancer (NSCLC), ${ }^{3,4}$ renal cell carcinoma (RCC), ${ }^{5}$ and melanoma. ${ }^{6}$ The anti-PD-L1 agent atezolizumab was recently approved for bladder cancer. ${ }^{7}$

Despite its impressive clinical benefits, checkpoint inhibition is associated with a series of inflammatory effects known as immune-related adverse events (irAEs). The irAEs, which include dermatologic, gastrointestinal, hepatic, endocrine, pulmonary, and other less common side effects, are believed to arise from immunologic enhancement and disruption of normal immune system homeostasis. They range from transient and benign to more severe and even fatal events in rare cases. Some studies have shown that the irAEs may be associated with clinical response to therapy. ${ }^{8}$ In a retrospective review of 119 patients with metastatic melanoma undergoing treatment with CTLA-4 inhibitors (tremelimumab or ipilimumab), 55\% of the patients who exhibited a
Department of Medicine, Feinberg School of Medicine, Northwestern University,

25I East Huron Street, Galter Suite

3-I50, Chicago, IL 606II, USA

$\mathrm{Tel}+\mathrm{I} 3129266895$

$\mathrm{Fax}+\mathrm{I} 3129260239$

Email benedito.carneiro@northwestern. edu 
radiographically evident irAE (colitis, arthritis, hypophysitis, thyroiditis, benign hilar lymphadenopathy, and myositis in this study) demonstrated clinical benefit, whereas only $10 \%$ of those without an overt irAE showed disease control. ${ }^{9}$

Among the irAEs, pneumonitis is rare but potentially life threatening. Three (2\%) patients died as a result of pneumonitis in a phase I study of nivolumab in NSCLC ${ }^{10}$ and one $(0.2 \%)$ patient died during another phase I trial of pembrolizumab in NSCLC. ${ }^{11}$ As such, pneumonitis has been identified as an event of special interest. ${ }^{12,13}$ There are limited data describing the incidence, characteristics, and sequelae of this adverse event (AE), but physicians should be mindful of the possibility of this diagnosis when patients receiving immunotherapy present with new respiratory complaints. This article reviews the epidemiology, presentation, diagnosis, and treatment of checkpoint inhibitor-related pneumonitis (CIP).

\section{Epidemiology}

Large clinical trials involving patients with advanced melanoma, NSCLC, and RCC have documented irAEs associated with the checkpoint inhibitors in $60-80 \%$ of patients. Severe irAEs (grade 3 or 4 ) occurred in $15-20 \%$ of patients. ${ }^{2,3,5,14-17}$ The PD-1 inhibitors tend to have a lower rate of irAEs compared with the CTLA-4 inhibitors, while the combination treatment has a higher rate of irAEs than either approach as monotherapy. The most common irAEs associated with checkpoint inhibitors include dermatologic (rash, pruritus), gastrointestinal (colitis, diarrhea), and endocrine (thyroiditis, hypophysitis) events. ${ }^{9,14,18}$

CIP is rare with an incidence of $<5 \%$ in clinical trials evaluating monotherapy ${ }^{15,16,19-21}$ and slightly $>5 \%$ in trials using combination therapy. ${ }^{22-24}$ Grade 3-4 pneumonitis (leading to hypoxia or respiratory compromise) is uncommon. Nonetheless, pneumonitis is one of the few irAEs that has been associated with drug-related deaths, so vigilance and awareness of this entity are critical. ${ }^{10-12}$

Unlike the majority of irAEs, pneumonitis seems to be less common with anti-CTLA-4 treatment than with anti-PD-1 treatment. ${ }^{12,16,19,25-27}$ Trials with ipilimumab, the majority of which have been conducted in patients with advanced melanoma, documented pneumonitis in $<1 \%$ of participants. $^{2,28,29}$ Isolated cases of life-threatening pneumonitis have been described, including in those receiving allogeneic hematopoietic cell transplantation. In contrast, a meta-analysis of 20 studies with PD-1 inhibitor treatment in melanoma, NSCLC, and RCC described a $2.7 \%$ incidence of pneumonitis for all grades and $0.8 \%$ for grade $\geq 3 .{ }^{22}$
Another study demonstrated that 43 of 915 patients $(5 \%)$ who received anti-PD-1/PD-L1 therapy developed pneumonitis. ${ }^{24}$ Pneumonitis was associated with treatment-related deaths in two early phase I trials. ${ }^{10,11}$ Table 1 summarizes the findings on CIP from the major trials of monotherapy to date.

Risk of pneumonitis and pneumonitis-related deaths in anti-PD-1 treatment may be dependent on tumor type. In the meta-analysis by Nishino et al, ${ }^{22}$ the incidence of pneumonitis was higher in NSCLC for all-grade (4.1\%) and grade $\geq 3$ $(0.8 \%)$ pneumonitis than in melanoma and was higher in $\mathrm{RCC}$ than in melanoma for all-grade pneumonitis but not for grade $\geq 3$. In contrast, other studies have shown that the rate of grades 3-4 pneumonitis is similar across tumor types, but with more treatment-related deaths due to pneumonitis in patients with NSCLC. ${ }^{10,11,30}$ It is unclear why NSCLC may be associated with more pneumonitis and/or treatment-related deaths, but a number of hypotheses (while not yet studied) seem plausible, including higher rates of pre-existing adverse pulmonary conditions (i.e., tobacco exposure, previous lung radiation) and previous exposure to drugs associated with interstitial lung disease, including taxanes, ${ }^{31}$ epidermal growth factor receptor tyrosine kinase inhibitors, ${ }^{32,33}$ and gemcitabine. ${ }^{34}$

Combination therapy is associated with higher rates of both all-grade and grade 3 or 4 pneumonitis than monotherapy $;^{22,23} \sim 10 \%$ of patients on combination therapy develop pneumonitis versus an average of $3 \%$ on monotherapy. ${ }^{24}$ The majority of studies of combination therapy have been conducted in patients with advanced melanoma. In contrast to the very low incidence of pneumonitis seen with any of the checkpoint inhibitors as monotherapy in patients with melanoma (Table 1), the incidence of all-grade and grade $\geq 3$ pneumonitis in combination therapy was reported to be $6.6 \%$ and $1.5 \%$, respectively. ${ }^{22}$ Patients treated with combination immunotherapy may be less likely to experience resolution of the irAE than patients treated with monotherapy. ${ }^{23}$

PD-L1 pathways are also an area of active investigation. Three studies of the PD-L1 inhibitor atezolizumab in NSCLC, RCC, and urothelial carcinoma documented incidence rates of all-grade pneumonitis of $2-3 \%$ without pneumonitis-related deaths. ${ }^{7,35,36}$ Data from a cohort of 43 patients suggested that there was no significant difference in pneumonitis incidence between anti-PD-1 and anti-PD-L1 monotherapy; ${ }^{24}$ however, larger data sets that include metaanalyses across tumor types are needed. Further studies will be needed to help elucidate the association between the PD-1/PD-L1 pathways and pneumonitis and to define its pathophysiology. 
Table I Incidence of pneumonitis in important clinical trials of checkpoint inhibitors in advanced malignancies

\begin{tabular}{|c|c|c|c|}
\hline Study & Drug & $\begin{array}{l}\text { Number of } \\
\text { patients (\%) } \\
\text { with all-grade } \\
\text { pneumonitis }\end{array}$ & $\begin{array}{l}\text { Number of } \\
\text { patients (\%) } \\
\text { with grade 3-4 } \\
\text { pneumonitis }\end{array}$ \\
\hline \multicolumn{4}{|l|}{ Melanoma } \\
\hline \multirow[t]{2}{*}{ Ribas et al ${ }^{51}$} & Pembrolizumab 2 mg/kg q3w & $3(2)$ & $0(0)$ \\
\hline & Pembrolizumab $10 \mathrm{mg} / \mathrm{kg}$ q3w & $3(2)$ & $2(1)$ \\
\hline \multirow[t]{3}{*}{ Robert et $\mathrm{al}^{20}$} & Pembrolizumab $10 \mathrm{mg} / \mathrm{kg}$ q2w & $0(0)$ & $0(0)$ \\
\hline & Pembrolizumab $10 \mathrm{mg} / \mathrm{kg} \mathrm{q3 \textrm {w }}$ & $0(0)$ & $0(0)$ \\
\hline & Ipilimumab 3 mg/kg q3w & $0(0)$ & $0(0)$ \\
\hline \multirow[t]{2}{*}{ Robert et al ${ }^{19}$} & Pembrolizumab 2 mg/kg q3w & $2(2)$ & $I(I)$ \\
\hline & Pembrolizumab $10 \mathrm{mg} / \mathrm{kg} \mathrm{q} 3 \mathrm{w}$ & $\mathrm{I}(\mathrm{I})$ & $0(0)$ \\
\hline Weber et $\mathrm{al}^{6}$ & Nivolumab $3 \mathrm{mg} / \mathrm{kg}$ q2w & $5(2)$ & $0(0)$ \\
\hline Robert et al ${ }^{16}$ & Nivolumab 3 mg/kg q2w & $3(2)$ & $0(0)$ \\
\hline Margolin et $\mathrm{al}^{29}$ & Ipilimumab 10 mg/kg q3w & $0(0)$ & $0(0)$ \\
\hline Hodi et $\mathrm{al}^{2}$ & Ipilimumab 3 mg/kg q3w & $0(0)$ & $0(0)$ \\
\hline \multirow[t]{3}{*}{ Wolchok et $\mathrm{al}^{28}$} & Ipilimumab $0.3 \mathrm{mg} / \mathrm{kg}$ q3w & $0(0)$ & $0(0)$ \\
\hline & Ipilimumab 3 mg/kg q3w & $0(0)$ & $0(0)$ \\
\hline & Ipilimumab 10 mg/kg q3w & $0(0)$ & $I(I)$ \\
\hline \multicolumn{4}{|l|}{ NSCLC } \\
\hline Borghaei et al ${ }^{17}$ & Nivolumab 3 mg/kg q2w & $4(I)$ & $3(1)$ \\
\hline Brahmer et $\mathrm{al}^{3}$ & Nivolumab 3 mg/kg q2w & $2(2)$ & $I(I)$ \\
\hline \multirow[t]{2}{*}{ Herbst et $\mathrm{al}^{30}$} & Pembrolizumab $2 \mathrm{mg} / \mathrm{kg} \mathrm{q} 3 \mathrm{w}$ & $14(4)$ & $6(2)$ \\
\hline & Pembrolizumab $10 \mathrm{mg} / \mathrm{kg}$ q3w & $12(4)$ & $6(2)$ \\
\hline Rizvi et al ${ }^{15}$ & Nivolumab $3 \mathrm{mg} / \mathrm{kg} \mathrm{q} 2 \mathrm{w}$ & $6(5)$ & $4(3)$ \\
\hline \multirow[t]{3}{*}{ Gettinger et $\mathrm{al}^{10}$} & Nivolumab I mg/kg q2w & $3(9)$ & $2(6)$ \\
\hline & Nivolumab 3 mg/kg q2w & I (3) & $0(0)$ \\
\hline & Nivolumab $10 \mathrm{mg} / \mathrm{kg} \mathrm{q} 2 \mathrm{w}$ & $4(7)$ & $\mathrm{I}(2)$ \\
\hline \multirow[t]{3}{*}{ Garon et al" } & Pembrolizumab 2 mg/kg q3w & $0(0)$ & Reported as 9 \\
\hline & Pembrolizumab $10 \mathrm{mg} / \mathrm{kg}$ q3w & $13(5)$ & $(2 \%)$ patients \\
\hline & Pembrolizumab $2 \mathrm{mg} / \mathrm{kg}$ q2w & $5(3)$ & $\begin{array}{l}\text { total, among all } \\
\text { groups }\end{array}$ \\
\hline \multicolumn{4}{|l|}{ RCC } \\
\hline Motzer et $\mathrm{al}^{5}$ & Nivolumab 3 mg/kg q2w & Not reported & Not reported \\
\hline \multicolumn{4}{|l|}{ Bladder cancer } \\
\hline Rosenberg et $\mathrm{al}^{7}$ & Atezolizumab $1200 \mathrm{mg}$ q3w & $7(2)$ & $2(1)$ \\
\hline
\end{tabular}

Abbreviations: NSCLC, non-small cell lung cancer; RCC, renal cell carcinoma; q3w, every 3 weeks; q2w, every 2 weeks.

\section{Diagnosis}

\section{Patient risk factors}

While certain risk factors - including advanced age, existing pulmonary lesions or decreased baseline respiratory function, history of pulmonary surgery, oxygen administration, and radiation exposure to the lung - are associated with a higher likelihood of developing drug-induced lung injury in general, the risk factors that predispose patients to developing pneumonitis after immunotherapy are not known. ${ }^{37}$ In a recent case series, $56 \%$ ( 24 of 43 ) of patients who developed pneumonitis on anti-PD-1/PD-L1 therapy were current or former smokers, while $44 \%$ of the patients were never smokers, but it is unclear whether smoking status represents a true risk factor. ${ }^{24}$ As described previously, NSCLC may be a risk factor for the development of pneumonitis and is more clearly a risk factor for pneumonitis-related death. ${ }^{22}$

\section{Symptoms}

The timing of onset and clinical manifestations of CIP can vary. The median onset appears to be 2.5 months after initiation of treatment; ${ }^{24,38}$ however, there is a wide range of 2-24 months. Some studies suggest that the onset may be later than the majority of irAEs. ${ }^{39,40}$ The onset does occur earlier with combination therapy versus monotherapy. ${ }^{24}$

Making the clinical diagnosis of CIP can be difficult given its resemblance to other conditions that may be encountered in cancer patients, such as worsening metastases and pulmonary infections. Symptoms of pneumonitis include nonproductive cough and unresolving dyspnea. ${ }^{24,25,39,41}$ Fever and chest pain are less common symptoms. Hypoxia may occur and progress rapidly leading to respiratory failure ${ }^{39}$ Interestingly, pneumonitis may appear on computed tomography (CT) scan before it becomes clinically evident. ${ }^{26}$ However, 
some patients with radiographically evident pneumonitis may remain asymptomatic. ${ }^{24,26}$ It is unknown whether the presence or absence of symptoms confers a difference in prognosis.

\section{Imaging}

Imaging is a key component of patient care in immunotherapy given its usefulness in assessing both treatment response and potential immune-related toxicity. All patients on checkpoint inhibitors who present with new respiratory complaints should undergo imaging of the chest, preferably crosssectional CT scan as radiography may fail to identify $\sim 25 \%$ of cases of pneumonitis, thus making it an inadequate diagnostic tool. ${ }^{24}$ Immunotherapy-related pneumonitis can present with varied radiographic findings. The most frequently reported findings are that of cryptogenic organizing pneumonia (COP), with ground-glass or consolidative opacities in peripheral or peribronchial distribution, followed by nonspecific interstitial pneumonia (NSIP), with ground-glass opacities and reticular opacities primarily in the peripheral and lower lungs. ${ }^{24-26,39}$ Pneumonitis presenting as acute interstitial pneumonia (AIP) and acute respiratory distress syndrome (ARDS) has also been reported, ${ }^{39}$ as has hypersensitivity pneumonitis (HP). ${ }^{24}$ In one study, AIP/ARDS manifested as the highest grade of lung injury followed by COP, while NSIP and HP had lower grade disease. ${ }^{38}$ Naidoo et $\mathrm{al}^{24}$ found that radiographic type actually evolved in two patients on PD-1 inhibitors, from COP-like to more nonspecific ground-glass opacities in one patient and from ground-glass opacities to interstitial infiltrates in another. Pleural effusions have also been reported. ${ }^{42}$

In addition to typical findings of pneumonitis, it is important to note that checkpoint inhibitors have also been associated with sarcoid-like pulmonary changes, including subpleural micronodular opacities and hilar lymphadenopathy. This form of lung injury has been reported with both CTLA-4 and PD-1 inhibition ${ }^{26,43-45}$ and, interestingly, has been associated with extrapulmonary granulomatous changes as well. Hilar lymphadenopathy can mimic progression of disease, and thus physicians should be aware of this potential pulmonary irAE.

\section{Pathology}

There is a paucity of data on the histopathologic findings of pneumonitis. One case report describes the autopsy findings of a 35-year-old female patient with metastatic melanoma treated with ipilimumab followed by nivolumab, who developed radiographic features of pneumonitis during ipilimumab treatment. ${ }^{46}$ Autopsy revealed diffuse alveolar damage as a probable contributing cause of death, as well as a sarcoid-like granulomatous reaction of the lung, and interstitial fibrosis. The pattern of granulomatous (sarcoid-like) inflammation has been reported with other drugs such as nitrofurantoin, sulfasalazine, and sirolimus. ${ }^{47,48}$ Naidoo et al $^{24}$ examined tissue samples from patients with CIP and found cellular interstitial pneumonitis, organizing pneumonia, and diffuse alveolar damage. Of note, some specimens did not show any abnormalities.

The tissue of patients with sarcoid-like pulmonary changes shows giant-cell granulomas with peripheral lymphocytes, with or without necrosis. ${ }^{43,45}$ Bronchoalveolar lavage (BAL) fluid of one patient showed increased lymphocytes $(58 \%)$ with an elevated CD4:CD8 ratio that is typical of sarcoidosis. ${ }^{43}$ The BAL fluid in patients with CIP has been reported to show lymphocytosis, ${ }^{25}$ but there are few reports and limited data. This remains an area of much needed study. ${ }^{25}$

\section{Treatment}

Establishing the diagnosis of immunotherapy-induced pneumonitis in a timely manner is imperative to allow prompt and appropriate administration of therapy. The optimal management of irAEs, summarized in Table 2, is based on clinical experience, case reports, and established management algorithms.

In clinical studies, toxicity severity is described using the Common Terminology Criteria for Adverse Events (CTCAE), which grades toxicities on a scale of 1 (mildest) to 5 (death related to that toxicity). For pneumonitis specifically, Grade 1 is defined by radiographic changes only, in the absence of symptoms. In these cases, it is recommended that delay of treatment be considered, and the patient should be monitored closely for symptoms every 2-3 days with repeated imaging at least every 3 weeks. ${ }^{23}$ Even though Bronstein et $\mathrm{al}^{9}$ showed that the presence of clinically silent but radiographically evident irAE may correlate with improved disease burden, none of the patients in this retrospective review were diagnosed with pneumonitis. Furthermore, prospective clinical trials are needed to determine whether checkpoint inhibitors may be safely continued in these patients. Steroids, however, are likely not needed in this clinical scenario.

Grade 2 pneumonitis is considered as a mild-to-moderate disease. Patients with grade 2 pneumonitis present with symptoms of mild-to-moderate severity, without new or worsening hypoxia and with radiographic infiltrates of varying appearance. Mild-to-moderate cases of pneumonitis are often managed successfully with steroids. ${ }^{23,39,40}$ We recommend intravenous methylprednisolone $0.5-1.0 \mathrm{mg} / \mathrm{kg}$ daily or the oral equivalent. ${ }^{40}$ Repeat imaging should be considered at the provider's discretion (between 1 and 3 days to every 
Table 2 Management of pneumonitis by grade

\begin{tabular}{|c|c|c|}
\hline $\begin{array}{l}\text { Grade of } \\
\text { pneumonitis }\end{array}$ & Symptoms & Management \\
\hline \multirow[t]{2}{*}{ Grade I } & None & Delay treatment \\
\hline & Radiographic changes only & Repeat imaging every 3 weeks \\
\hline \multirow[t]{5}{*}{ Grade 2} & Mild to moderate & Delay treatment \\
\hline & Dyspnea and cough & Consider admission to hospital \\
\hline & & Methylprednisolone IV 0.5-I.0 mg/kg/day \\
\hline & & Taper steroids over I month \\
\hline & & Repeat imaging in days to weeks \\
\hline \multirow[t]{6}{*}{ Grade 3-4 } & Severe & Delay treatment, consider permanent cessation \\
\hline & Hypoxia & Admit to hospital or ICU \\
\hline & Life-threatening respiratory & Methylprednisolone IV $2-4$ mg/kg/day \\
\hline & compromise & Consider additional immunosuppression at 48 hours \\
\hline & & Taper steroids over 6 weeks \\
\hline & & Repeat imaging in days to weeks \\
\hline
\end{tabular}

Abbreviations: IV, intravenous; ICU, intensive care unit.

few weeks) to ensure improvement. The time to resolution of mild-to-moderate pneumonitis is reported at anywhere from 2 to 8 weeks. ${ }^{15,39}$ Furthermore, steroids for the treatment of irAEs do not seem to interfere with the efficacy of immunotherapy once an irAE has occured, ${ }^{49,50}$ but further studies are needed to confirm this observation. When symptoms return to near baseline, steroids should be tapered over $\sim 1$ month. Once symptoms and radiographic changes have resolved, checkpoint inhibition may be resumed. ${ }^{39}$ If symptoms do not improve after 2 weeks or are worsening, pneumonitis should be treated as grade $3-4$.

Patients with grade 3-4 pneumonitis have significant symptoms, new or worsening hypoxia, or life-threatening respiratory compromise. They should be admitted to the hospital or intensive care unit. Bronchoscopy and other appropriate diagnostic studies should be performed to exclude infectious or alternative etiologies prior to starting more aggressive intravenous methylprednisolone that should be initiated at a dose of $2-4 \mathrm{mg} / \mathrm{kg}$ /day or parental equivalent. If symptoms improve to baseline, steroids should be tapered over at least 6 weeks. However, if symptoms are not improving or worsening after 48 hours, additional immunosuppression should be considered, including infliximab, cyclophosphamide, intravenous immunoglobulin (IVIG), and mycophenolate mofetil. ${ }^{23,39}$ Patients who experience moderate-to-severe pneumonitis should not receive further doses of checkpoint inhibitor. ${ }^{40}$

\section{Conclusion}

The introduction of the immune checkpoint inhibitors has reinvigorated the management of a number of malignancies. Anti-CTLA-4 and anti-PD-1 agents have demonstrated marked efficacy in the treatment of advanced cancers and have shed light on new tumor biologic and immunologic principles. Nonetheless, immunotherapy is associated with rare but potentially life-threatening irAEs. The widespread use of these agents demands a greater understanding of the clinical manifestations, diagnosis, and treatment of these side effects. Pneumonitis is an irAE of special interest, as a result of three pneumonitis-related deaths in a phase I study of nivolumab in NSCLC. ${ }^{10}$ The adverse event is rare but life-threatening, with varied presenting symptoms and radiographic manifestations. While COP and NSIP are currently the most commonly reported radiographic findings, AIP, ARDS, and HP have also been reported in the literature. Health care workers must be mindful of the multiple possible clinical presentations of this irAE, as well as the current guidelines regarding treatment. Early recognition and prompt initiation of steroids is critical to improve outcome, with additional immunosuppressive agents reserved for more severe cases.

\section{Acknowledgment}

This study is supported by The Woman's Board of Northwestern Memorial Hospital and the Developmental Therapeutics Program, Division of Hematology/Oncology.

\section{Disclosure}

The authors report no conflicts of interest in this work.

\section{References}

1. Buchbinder EI, Desai A. CTLA-4 and PD-1 pathways: similarities, differences, and implications of their inhibition. Am J Clin Oncol. 2016;39(1):98-106.

2. Hodi FS, O'Day SJ, McDermott DF, et al. Improved survival with ipilimumab in patients with metastatic melanoma. $N$ Engl J Med. 2010;363(8):711-723. 
3. Brahmer J, Reckamp KL, Baas P, et al. Nivolumab versus docetaxel in advanced squamous-cell non-small-cell lung cancer. $N$ Engl J Med. 2015;373(2):123-135.

4. Herbst RS, Baas P, Kim D-W, et al. Pembrolizumab versus docetaxel for previously treated, PD-L1-positive, advanced non-small-cell lung cancer (KEYNOTE-010): a randomised controlled trial. Lancet. 2016;387(10027):1540-1550.

5. Motzer RJ, Escudier B, McDermott DF, et al. Nivolumab versus everolimus in advanced renal-cell carcinoma. $N$ Engl J Med. 2015;373(19):1803-1813.

6. Weber JS, D'Angelo SP, Minor D, et al. Nivolumab versus chemotherapy in patients with advanced melanoma who progressed after anti-CTLA-4 treatment (CheckMate 037): a randomised, controlled, open-label, phase 3 trial. Lancet Oncol. 2015;16(4):375-384.

7. Rosenberg JE, Hoffman-Censits J, Powles T, et al. Atezolizumab in patients with locally advanced and metastatic urothelial carcinoma who have progressed following treatment with platinum-based chemotherapy: a single-arm, multicentre, phase 2 trial. Lancet. 2016;387(10031):1909-1920.

8. Downey SG, Klapper JA, Smith FO, et al. Prognostic factors related to clinical response in patients with metastatic melanoma treated by CTL-associated antigen-4 blockade. Clin Cancer Res. 2007;13(22 pt 1):6681-6688.

9. Bronstein Y, Ng CS, Hwu P, et al. Radiologic manifestations of immune-related adverse events in patients with metastatic melanoma undergoing anti-CTLA-4 antibody therapy. Am J Roentgenol. 2011;197(6):W992-W1000.

10. Gettinger SN, Horn L, Gandhi L, et al. Overall survival and long-term safety of nivolumab (anti-programmed death 1 antibody, BMS-936558, ONO-4538) in patients with previously treated advanced non-small-cell lung cancer. J Clin Oncol. 2015;33(18):2004-2012.

11. Garon EB, Rizvi NA, Hui R, et al. Pembrolizumab for the treatment of non-small-cell lung cancer. N Engl J Med. 2015;372(21): 2018-2028.

12. Topalian SL, Hodi FS, Brahmer JR, et al. Safety, activity, and immune correlates of anti-PD-1 antibody in cancer. $N$ Engl $\mathrm{J} \mathrm{Med}$. 2012;366(26):2443-2454.

13. Topalian SL, Sznol M, McDermott DF, et al. Survival, durable tumor remission, and long-term safety in patients with advanced melanoma receiving nivolumab. J Clin Oncol. 2014;32(10):1020-1030.

14. Weber JS, Dummer R, de Pril V, et al. Patterns of onset and resolution of immune-related adverse events of special interest with ipilimumab. Cancer. 2013;119(9):1675-1682.

15. Rizvi NA, Mazières J, Planchard D, et al. Activity and safety of nivolumab, an anti-PD-1 immune checkpoint inhibitor, for patients with advanced, refractory squamous non-small-cell lung cancer (CheckMate 063): a phase 2, single-arm trial. Lancet Oncol. 2015;16(3):257-265.

16. Robert C, Long GV, Brady B, et al. Nivolumab in previously untreated melanoma without BRAF mutation. $N$ Engl J Med. 2015;372(4):320-330.

17. Borghaei H, Paz-Ares L, Horn L, et al. Nivolumab versus docetaxel in advanced nonsquamous non-small-cell lung cancer. $N$ Engl J Med. 2015;373(17):1627-1639.

18. Costa R, Carneiro BA, Agulnik M, et al. Toxicity profile of approved anti-PD-1 monoclonal antibodies in solid tumors: a systematic review and meta-analysis of randomized clinical trials. Oncotarget. 2017;8(5):8910-8920.

19. Robert C, Ribas A, Wolchok JD, et al. Anti-programmed-death-receptor-1 treatment with pembrolizumab in ipilimumab-refractory advanced melanoma: a randomised dose-comparison cohort of a phase 1 trial. Lancet. 2014;384(9948):1109-1117.

20. Robert C, Schachter J, Long GV, et al. Pembrolizumab versus ipilimumab in advanced melanoma. N Engl J Med. 2015;372(26):2521-2532.

21. Larkin J, Chiarion-Sileni V, Gonzalez R, et al. Combined nivolumab and ipilimumab or monotherapy in untreated melanoma. N Engl J Med. 2015;373(1):23-34.
22. Nishino M, Giobbie-Hurder A, Hatabu H, et al. Incidence of programmed cell death 1 inhibitor-related pneumonitis in patients with advanced cancer: a systematic review and meta-analysis. JAMA Oncol. 2016;2(12): 1607-1616.

23. Postow MA, Chesney J, Pavlick AC, et al. Nivolumab and ipilimumab versus ipilimumab in untreated melanoma. NEngl J Med. 2015;372(21): 2006-2017.

24. Naidoo J, Wang X, Woo KM, et al. Pneumonitis in patients treated with anti-programmed death-1/programmed death ligand 1 therapy. J Clin Oncol. 2017;35(7):709-717.

25. Barjaktarevic IZ, Qadir N, Suri A, Santamauro JT, Stover D. Organizing pneumonia as a side effect of ipilimumab treatment of melanoma. Chest. 2013;143(3):858-861.

26. Tirumani SH, Ramaiya NH, Keraliya A, et al. Radiographic profiling of immune-related adverse events in advanced melanoma patients treated with ipilimumab. Cancer Immunol Res. 2015;3(10):1185-1192.

27. Hamid O, Robert C, Daud A, et al. Safety and tumor responses with lambrolizumab (Anti-PD-1) in melanoma. N Engl J Med. 2013;369(2): 134-144.

28. Wolchok JD, Neyns B, Linette G, et al. Ipilimumab monotherapy in patients with pretreated advanced melanoma: a randomised, double-blind, multicentre, phase 2, dose-ranging study. Lancet Oncol. 2010;11(2): 155-164.

29. Margolin K, Ernstoff MS, Hamid O, et al. Ipilimumab in patients with melanoma and brain metastases: an open-label, phase 2 trial. Lancet Oncol. 2012;13(5):459-465.

30. Nagata S, Ueda N, Yoshida Y, Matsuda H, Maehara Y. Severe interstitial pneumonitis associated with the administration of taxanes. $J$ Infect Chemother. 2010;16(5):340-344.

31. Masahiko A, Isamu O, Nobuyuki Y, et al. Predictive factors for interstitial lung disease, antitumor response, and survival in nonsmall-cell lung cancer patients treated with gefitinib. J Clin Oncol. 2006;24(16):2549-2556.

32. Takano T, Ohe Y, Kusumoto M, et al. Risk factors for interstitial lung disease and predictive factors for tumor response in patients with advanced non-small cell lung cancer treated with gefitinib. Lung Cancer. 2004;45(1):93-104.

33. Gupta N, Ahmed I, Steinberg H, et al. Gemcitabine-induced pulmonary toxicity: case report and review of the literature. Am J Clin Oncol. 2002;25(1):96-100.

34. Fehrenbacher L, Spira A, Ballinger M, et al. Atezolizumab versus docetaxel for patients with previously treated non-small-cell lung cancer (POPLAR): a multicentre, open-label, phase 2 randomised controlled trial. Lancet. 2016;387(10030):1837-1846.

35. McDermott DF, Sosman JA, Sznol M, et al. Atezolizumab, an anti-programmed death-ligand 1 antibody, in metastatic renal cell carcinoma: long-term safety, clinical activity, and immune correlates from a phase Ia study. J Clin Oncol. 2016;34(8):833-842.

36. Kubo K, Azuma A, Kanazawa M, et al. Consensus statement for the diagnosis and treatment of drug-induced lung injuries. Respir Investig. 2013;51(4):260-277.

37. Nishino M, Ramaiya NH, Awad MM, et al. PD-1 inhibitor-related pneumonitis in advanced cancer patients: radiographic patterns and clinical course. Clin Cancer Res. 2016;22(24):6051-6060.

38. Nishino M, Sholl LM, Hatabu H, et al. Anti-PD-1-related pneumonitis during cancer immunotherapy. N Engl J Med. 2015;373(3):288-290.

39. Friedman CF, Proverbs-Singh TA, Postow MA. Treatment of the immune-related adverse effects of immune checkpoint inhibitors: a review. JAMA Oncol. 2016;2(10):1346-1353.

40. Mis L, Clarke JM. Ipilimumab-induced pneumonitis: a case report. J Pharm Technol. 2013;29:94-98.

41. Kolla BC, Patel MR. Recurrent pleural effusions and cardiac tamponade as possible manifestations of pseudoprogression associated with nivolumab therapy- a report of two cases. J Immunother Cancer. 2016;4:80.

42. Berthod G, Lazor R, Letovanec I, et al. Pulmonary sarcoid-like granulomatosis induced by ipilimumab. J Clin Oncol. 2012;30(17):e156-e159. 
43. Bashey A, Medina B, Corringham S, et al. CTLA4 blockade with ipilimumab to treat relapse of malignancy after allogeneic hematopoietic cell transplantation. Blood. 2009;113(7):1581-1588.

44. Montaudié H, Pradelli J, Passeron T, Lacour JP, Leroy S. Pulmonary sarcoid-like granulomatosis induced by nivolumab. $\mathrm{Br} J$ Dermatol. 2017;176(4):1060-1063.

45. Danlos F-X, Pagès C, Baroudjian B, et al. Nivolumab-induced sarcoidlike granulomatous reaction in a patient with advanced melanoma. Chest. 2016;149(5):e133-e136.

46. Koelzer VH, Rothschild SI, Zihler D, et al. Systemic inflammation in a melanoma patient treated with immune checkpoint inhibitors - an autopsy study. J Immunother Cancer. 2016;4:13.

47. Garrean S, Massad MG, Tshibaka M, Hanhan Z, Caines AE, Benedetti E. Sirolimus-associated interstitial pneumonitis in solid organ transplant recipients. Clin Transplant. 2005;19(5):698-703.
48. Mohyuddin GR, Sultan F, Zhang K, Khaleeq G. Sulfasalazine induced lung toxicity masquerading as sarcoidosis - case report and review of the literature. Sarcoidosis Vasc Diffuse Lung Dis. 2013;30(3):226-230.

49. Troy ZH, Nelly GA, Thu-Oanh D, et al. Immune-related adverse events, need for systemic immunosuppression, and effects on survival and time to treatment failure in patients with melanoma treated with ipilimumab at memorial Sloan Kettering Cancer Center. J Clin Oncol. 2015;33(28):3193-3198.

50. Weber JS, Antonia SJ, Topalian SL, et al. Safety profile of nivolumab (NIVO) in patients (pts) with advanced melanoma (MEL): a pooled analysis. ASCO Annu Meet Proc. 2015;33:9018.

51. Ribas A, Puzanov I, Dummer R, et al. Pembrolizumab versus investigator-choice chemotherapy for ipilimumab-refractory melanoma (KEYNOTE-002): a randomised, controlled, phase 2 trial. Lancet Oncol. 2015;16(8):908-918.
Cancer Management and Research

\section{Publish your work in this journal}

Cancer Management and Research is an international, peer-reviewed open access journal focusing on cancer research and the optimal use of preventative and integrated treatment interventions to achieve improved outcomes, enhanced survival and quality of life for the cancer patient The manuscript management system is completely online and includes

\section{Dovepress}

a very quick and fair peer-review system, which is all easy to use. Visit http://www.dovepress.com/testimonials.php to read real quotes from published authors. 